\section{(2) OPEN ACCESS}

\title{
Immunogenicity and safety of anti-SARS-CoV-2 mRNA vaccines in patients with chronic inflammatory conditions and immunosuppressive therapy in a monocentric cohort
}

\author{
Ulf M Geisen, ${ }^{1}$ Dennis K Berner, ${ }_{1}^{1}$ Florian Tran, ${ }^{2,3}$ Melike Sümbül, ${ }_{1}^{4}$ Lena Vullriede, ${ }^{1}$ \\ Maria Ciripoi, ${ }^{1}$ Hayley M Reid, ${ }^{1}$ Annika Schaffarzyk, ${ }^{5}$ Ann C Longardt, ${ }^{6}$ \\ Jeanette Franzenburg, ${ }^{7,8}$ Paula Hoff, ${ }^{9}$ Jan $\mathrm{H}$ Schirmer, ${ }^{1}$ Rainald Zeuner, ${ }^{1}$ \\ Anette Friedrichs, ${ }^{2}$ Andrea Steinbach, ${ }^{1}$ Christine Knies, ${ }^{10}$ Robert DH Markewitz (1) , ${ }^{11}$ \\ Peter J Morrison, ${ }^{4}$ Sascha Gerdes, ${ }^{4}$ Stefan Schreiber, ${ }^{7,12}$ Bimba F Hoyer (1) ${ }^{1}$
}

\begin{abstract}
Handling editor Josef $S$ Smolen

- Additional material is published online only. To view please visit the journal online (http://dx.doi.org/10.1136/ annrheumdis-2021-220272).
\end{abstract}

For numbered affiliations see end of article.

\section{Correspondence to} Professor Bimba F Hoyer, Medical Department I, Department for Rheumatology and Clinical Immunology, University Medical Center Schleswig-Holstein Campus Kiel, Kiel, Schleswig-Holstein, Germany;

Bimba.Hoyer@uksh.de

SS and BFH are joint senior authors.

Received 3 March 2021 Revised 10 March 2021 Accepted 12 March 2021 Published Online First 24 March 2021

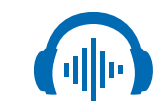

Listen to Podcast ard.bmj.com

Check for updates

(C) Author(s) (or their employer(s)) 2021. Re-use permitted under CC BY-NC. No commercial re-use. See rights and permissions. Published by BMJ.

\begin{tabular}{l}
\hline To cite: Geisen UM, \\
Berner DK, Tran F, \\
et al. Ann Rheum Dis \\
2021;80:1306-1311. \\
\hline
\end{tabular}

\section{ABSTRACT}

Introduction In light of the SARS-CoV-2 pandemic, protecting vulnerable groups has become a high priority. Persons at risk of severe disease, for example, those receiving immunosuppressive therapies for chronic inflammatory cdiseases (CIDs), are prioritised for vaccination. However, data concerning generation of protective antibody titres in immunosuppressed patients are scarce. Additionally, mRNA vaccines represent a new vaccine technology leading to increased insecurity especially in patients with CID.

Objective Here we present for the first time, data on the efficacy and safety of anti-SARS-CoV-2 mRNA vaccines in a cohort of immunosuppressed patients as compared with healthy controls.

Methods 42 healthy controls and 26 patients with CID were included in this study (mean age 37.5 vs 50.5 years). Immunisations were performed according to national guidelines with mRNA vaccines. Antibody titres were assessed by ELISA before initial vaccination and 7 days after secondary vaccination. Disease activity and side effects were assessed prior to and 7 days after both vaccinations.

Results Anti-SARS-CoV-2 antibodies as well as neutralising activity could be detected in all study participants. IgG titres were significantly lower in patients as compared with controls (2053 binding antibody units $(\mathrm{BAU}) / \mathrm{mL} \pm 1218$ vs $2685 \pm 1102)$. Side effects were comparable in both groups. No severe adverse effects were observed, and no patients experienced a disease flare.

Conclusion We show that SARS-CoV-2 mRNA vaccines lead to development of antibodies in immunosuppressed patients without considerable side effects or induction of disease flares. Despite the small size of this cohort, we were able to demonstrate the efficiency and safety of mRNA vaccines in our cohort.

\section{INTRODUCTION}

The SARS-CoV-2 pandemic continues to threaten the health of patients worldwide. Patients receiving immunosuppressive medication, for example, in the context of transplantation or chronic inflammatory diseases (CID), are considered to be at a

\section{Key messages}

What is already known about this subject?

- Data on the efficacy and safety of mRNA vaccines in patients with immunosuppressive therapies is not available so far.

What does this study add?

- In our cohort, mRNA vaccines against SARSCoV-2 showed a considerable immunogenicity in patients.

- Side effects in patients were comparable with controls with systemic side effects being less frequent.

- No flares of the underlying inflammatory condition could be observed in the context of the vaccination.

How might this impact on clinical practice or future developments?

- The data in this study indicate that mRNA vaccines against SARS-CoV-2 are immunogenic and safe in patients with chronic inflammatory diseases.

higher risk of severe manifestations of COVID19. Generally, patients receiving immunosuppression are considered to have an increased risk for infections. However, registry data appear to indicate that in the context of SARS-CoV-2 not every immunosuppressed patient has an increased risk of severe COVID-19. Indeed, biological therapies have been identified as decreasing the risk for hospitalisation due to COVID-19 in cohorts of patients with rheumatic diseases, chronic inflammatory bowel diseases and psoriasis. ${ }^{1-5}$ The most important factors associated with a higher risk of hospitalisation and death across multiple indications and forms of immunosuppression were found to be older age, high underlying disease activity as well as high glucocorticoid dosages (at dosages equivalent to prednisolone $\geq 10 \mathrm{mg}$ ). ${ }^{167}$ Additionally, B cell depleting drugs, that is, rituximab, might represent a risk factor. ${ }^{8}$ Until now, there is 
insufficient registry data for other drugs commonly used to treat patients with CID in terms of increased risk of severe COVID-19. ${ }^{1910}$ However, patients have minimised their risk by sheltering in place early and reducing infection contacts (own unpublished data).

Several drugs used in the management of CID have been analysed as potential treatments for COVID-19, especially in attenuating the so-called cytokine storm, some of which have shown considerable benefit. ${ }^{11}$

Vaccination against SARS-CoV-2 is now a reality for the most vulnerable and continues to spread to encompass patients receiving immunosuppressive therapies. However, patients with a higher risk being older, taking more steroids and having high underlying disease activity are known to respond less to vaccines. ${ }^{12-15}$ Additionally, patients with CID and those taking anticytokine therapies or immunosuppression were excluded from the phase III trials for all vaccines approved by the European Medicins Agency (EMA) and US Food and Drug Administration (FDA). ${ }^{16-18}$

The scarce data available on vaccine response under immunosuppression for other vaccines leaves many open questions in relation to SARS-CoV-2 vaccination. ${ }^{15} 19$

As shown for several vaccines in patients with chronic inflammatory diseases and transplanted patients, antibody titres postvaccination may be decreased depending on the vaccine and the treatment (although this is not always the case). ${ }^{151920}$ In relation to SARS-CoV-2, it is currently unclear how immunosuppression for CID affects vaccine response. There are also additional concerns regarding reactivation of the inflammatory disease by new mRNA vaccines.

We therefore provide for the first-time data comparing the immunogenicity and safety of SARS-CoV-2 mRNA vaccines in patients with CID undergoing immunosuppressive therapy compared with healthy controls in a monocentric observational study.

\section{MATERIAL AND METHODS}

Healthy individuals as well as the majority of patients with CID were recruited from healthcare workers of the University Medical Center in Kiel and other surrounding hospitals. Aged patients were recruited from the patient cohort of the rheumatology outpatient department in Kiel. Healthy controls and patients were vaccinated based on the occupational exposure risk or age associated risk at official vaccination centres. The vaccination was not part of the study.

Forty-two healthy controls and 26 patients with CID were enrolled into this non-randomised trial. All volunteers were eligible for early vaccination according to German federal regulations and received mRNA vaccines from either BioNtech/Pfizer or Moderna. Five Patients were immunised with COVID-19 vaccine Moderna; all others received Comirnaty. Vaccines were given to the participants with an interval of 35 days between the two doses. Patients older than 80 years were immunised twice with a 21-day interval.

Monitoring for disease activity (disease activity score 28 (DAS28), Patients Global Assessment (PGA) and Physician Global Assessment (PhGA)) was performed at baseline (before first vaccination), 7 days after the first vaccination, on the day of the second immunisation and 7 days thereafter plus any other time point in between if disease flares were experienced. Routine laboratory monitoring was performed at each time point. Side effects were monitored by online surveys and medical history taking 14 days after secondary vaccination.
IgG antibodies against SARS-CoV-2 were quantified by ELISA according to manufacturer's protocol (EUROIMMUN QuantiVac), and neutralising antibodies were measured using an ELISA-based neutralisation test system according to manufacturer's protocol (cPass system, kindly provided by medac). ${ }^{21}$ Additionally, anti-SARS-CoV-2 IgA titres were quantified according to manufacturer's instruction (Aeskulisa, Aeskulap)

Antibody testing were normally performed on day 0 , the day of secondary immunisation and day 7 after secondary immunisation.

Statistical analysis was performed using GraphPad Prism. Mann-Whitney tests was used for statistical analysis, and $p$ values below 0.05 were considered significant.

\section{RESULTS}

\section{Demographics}

Healthy controls were $69.2 \%$ women with a mean age of 37.5 years ( \pm 13.4 ; range $22-61)$. All were healthcare professionals. No participant previously had SARS-CoV-2-infection before vaccination.

The CID patient cohort consisted of $64.3 \%$ women with a mean age of 50.5 years $( \pm 15.8$; range $24-89)$. Again, the majority were healthcare workers and none had been infected with SARS-CoV-2 prior to vaccination. Table 1 contains detailed CID patient information (inflammatory diseases and therapies).

\section{SARS-CoV-2 mRNA vaccines show immunogenicity in patients with CID}

Neutralising antibodies and total anti-SARS-CoV-2 IgG were detected in all patients with CID and healthy controls after the second vaccination. No non-responders were detected in any group. None of the participants displayed considerable antibody titres before the first vaccination, indicating no prior infection.

While the healthy control group showed a mean anti-SARSCoV-2-IgG titre of $2685 \mathrm{BAU} / \mathrm{mL}$ ( \pm 1102 , 793-3840), patients with CID exhibited significantly lower levels of specific immunoglobulins against the SARS-CoV-2 spike protein (mean 2053 $\mathrm{BAU} / \mathrm{mL} \pm 1218,98.2-3840) 7$ days after the secondary immunisation $(p=0.037)$. Nevertheless, all patients presented with an antibody titre above the ELISA cut-off (figure $1 \mathrm{~A}$ and $\mathrm{B}$ ). When comparing groups by age range however, this difference was not significant anymore (figure $1 \mathrm{G}$ ).

Patients with CID also had lower levels of neutralising antibodies, with a mean inhibitory activity level of $96.04 \%$ detected in healthy controls $( \pm 1.551,91-97)$, whereas patients presented with a mean inhibitory level of $87.42 \%( \pm 17.94,37-97$; $\mathrm{p}=0.0442$ ) (figure 1C, D and $\mathrm{H}$ ).

Of interest, SARS-CoV-2 IgA antibodies were detectable in nearly all patients and healthy controls 7 days after secondary immunisation. Again, patients with CID had lower specific IgA levels compared with healthy controls (mean $24.52 \pm 30.48 \mathrm{U} / \mathrm{mL}$ vs $47.65 \pm 45.12 \mathrm{U} / \mathrm{mL} ; \mathrm{p}=0.0035)$. One patient with CID had no detectable specific IgA, while an additional two patients and three healthy controls showed IgA levels below the cut-off (E and F).

Comparing the largest therapeutic groups (TNF blockade vs conventional disease-modifying antirheumatic drugs (cDMARDs) vs anti-interleukin 17) showed no significant difference between those therapies (online supplemental figure 1).

\section{Patients with CID had a marginal propensity towards mild} vaccine side effects compared with healthy controls

Side effects as documented by an online survey were comparable in both groups. Mild systemic side effects such as fatigue and myalgia were more frequent in the CID patient cohort relative 
Table 1 Demographics and clinical characteristics of the included patients

\begin{tabular}{|c|c|c|c|c|c|}
\hline Sex & $\begin{array}{l}\text { Age } \\
\text { (years) }\end{array}$ & Inflammatory disease & $\begin{array}{l}\text { Biological } \\
\text { DMARD }\end{array}$ & Conventional DMARD & Steroids \\
\hline $\mathrm{F}$ & 44 & Psoriatic arthritis & Golimumab & Leflunomide & $5 \mathrm{mg}$ prednisolone \\
\hline $\mathrm{F}$ & 35 & Psoriatic arthritis & Certolizumab pegol & - & - \\
\hline $\mathrm{F}$ & 43 & Rheumatoid arthritis & Certolizumab pegol & - & $5 \mathrm{mg}$ prednisolone \\
\hline M & 46 & MCTD & - & Hydroxychloroquine & - \\
\hline $\mathrm{F}$ & 39 & Rheumatoid arthritis & Etanercept & Leflunomide & - \\
\hline $\mathrm{F}$ & 51 & Rheumatoid arthritis & - & Sulfasalazine & - \\
\hline $\mathrm{F}$ & 65 & Spondyloarthropathy & Infliximab & - & - \\
\hline M & 38 & Spondyloarthropathy & Etanercept & - & - \\
\hline $\mathrm{F}$ & 45 & Sarcoidosis & Infliximab & - & $15 \mathrm{mg}$ prednisolone \\
\hline $\mathrm{F}$ & 33 & Rheumatoid arthritis & Certolizumab pegol & - & - \\
\hline M & 84 & Giant cell vasculitis & Tocilizumab & - & $5 \mathrm{mg}$ prednisolone \\
\hline $\mathrm{F}$ & 47 & Psoriasis & Ixekizumab & - & - \\
\hline M & 83 & Rheumatoid arthritis & Etanercept & - & $2.5 \mathrm{mg}$ prednisolone \\
\hline M & 38 & Crohn's disease & Vedolizumab & - & - \\
\hline $\mathrm{F}$ & 53 & Rheumatoid arthritis & - & Leflunomide & $7 \mathrm{mg}$ prednisolone \\
\hline $\mathrm{F}$ & 24 & Systemic lupus erythematosus & - & Hydroxychloroquine & - \\
\hline M & 42 & Psoriasis & Adalimumab & - & - \\
\hline $\mathrm{F}$ & 54 & Rheumatoid arthritis & Adalimumab & - & - \\
\hline M & 58 & Spondyloarthropathy & Secukinumab & - & - \\
\hline $\mathrm{F}$ & 51 & Psoriasis & Secukinumab & - & - \\
\hline $\mathrm{F}$ & 53 & Crohn's disease & Infliximab & - & - \\
\hline M & 61 & Psoriasis & Ustekinumab & - & - \\
\hline M & 36 & Systemic lupus erythematosus & Belimumab & Hydroxychloroquine & - \\
\hline $\mathrm{F}$ & 89 & Myositis & - & - & $2.5 \mathrm{mg}$ prednisolone \\
\hline $\mathrm{F}$ & 49 & Multiple sclerosis/Crohn's disease & - & Azathioprine & - \\
\hline $\mathrm{F}$ & 54 & Rheumatoid arthritis & Adalimumab & - & - \\
\hline
\end{tabular}

to healthy controls (53.8\% vs $43.2 \%$ and $42.3 \%$ vs $31.6 \%)$. A similar pattern was seen for headache (38.5\% vs $35.1 \%)$. Fever was completely absent in patients with CID while being reported by $13.5 \%$ of the healthy cohort. Arthralgia was comparable in both groups.

Some additional side effects were reported in both groups such as nausea and vomiting, thoracic pain and exacerbation of pre-existing asthma (table 2). However, not all controls did report side effects.

\section{Inflammatory disease activity remained stable throughout the study}

Activity of inflammatory disease was monitored by DAS28 for patients with inflammatory arthritis and PGA as well as PhGA for all patients with CID.

We did not observe any inflammatory arthritis flares (delta DAS28 >0.6) in the context of either vaccination time points. Delta PGA and PhGA showed a maximal mean change of 0.4 $( \pm 1.29)$ at the time point of the secondary vaccination, whereas the delta for the last time point ( 7 days after secondary vaccination) was $0.076( \pm 0.4)$ compared with baseline. No patient with CID needed to adjust DMARD or glucocorticoid therapy in the 6 weeks of trial duration (figure 2A,B).

\section{DISCUSSION}

Due to the ongoing COVID-19 pandemic, the effectiveness and safety of novel mRNA vaccines in immunosuppressed patients is under discussion, but real-world data have been missing. Patients with CID as well as physicians have been confronted with the question as to whether immunosuppressed patients, who were excluded from the phase III vaccine trials, should be vaccinated without prior knowledge of the potential risks of adverse events and changes in efficacy when this new type of vaccine is used in patients with CID. This lack of information has created additional insecurity and hesitation in both physicians and patients.

With the data acquired in this investigation, we are able to demonstrate for the first time in a mixed cohort of patients with CID undergoing a spectrum of immunosuppressive treatments that such conditions, and therapies do not significantly abrogate the anti-SARS-CoV-2 antibody response after vaccination. Hence, in this cohort, no patient with CID was a complete non-responder even though antibody titres were slightly lower in patients with CID compared with controls. Furthermore, all patients had considerable levels of neutralising antibodies 7 days after secondary vaccination. Moreover, the thee patients with CID and three healthy controls with low IgA serum levels displayed substantial neutralisation capacity and IgG levels. Nevertheless, a direct comparison with phase III study data is not possible as different testing systems were used. ${ }^{22}$ The only patient with a very low IgG level and absent IgA response was an 85 -year-old patient with multiple comorbidities, known to influence vaccine response additionally, receiving anti-interleukin 6 therapy and glucocorticoids. Therefore, age-related immunosenesence may also contribute to the low Ig levels. Nevertheless, this patient also mounted a significant neutralising response after vaccination. Regarding the age difference between patients and controls, the overall antibody levels showed a significant difference between both groups. When comparing the according age groups, however, differences in antibody levels were not found to be significant.

A fraction of patients paused their DMARD medication around the vaccinations. In this cohort, no effect of pausing versus continuing was observed in our cohort. The same holds true for the use of non-steroidal anti-inflammatory drugs. However, none of the patients was in methotrexate therapy, which has been reported to have an impact on vaccination 
A

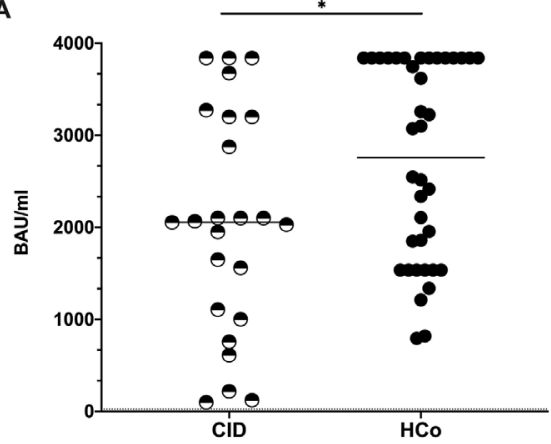

C
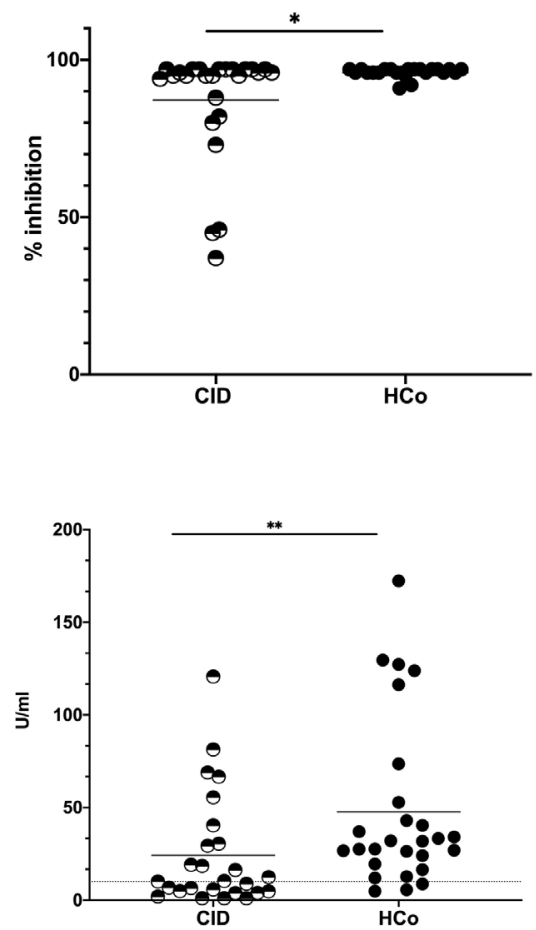

G

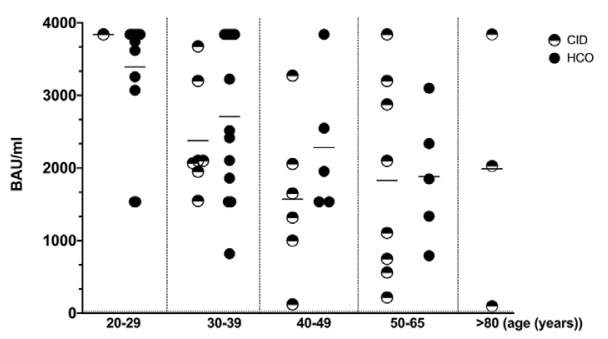

B

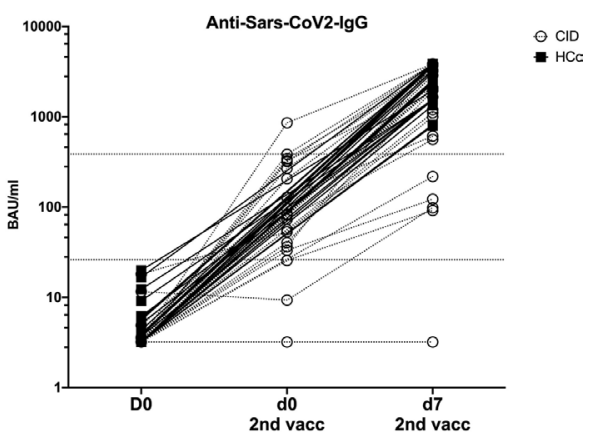

D

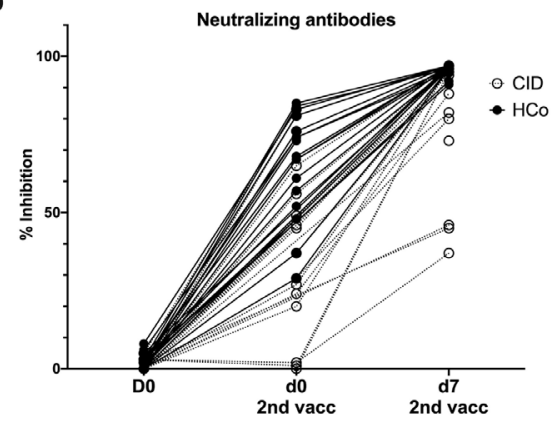

$\mathbf{F}$

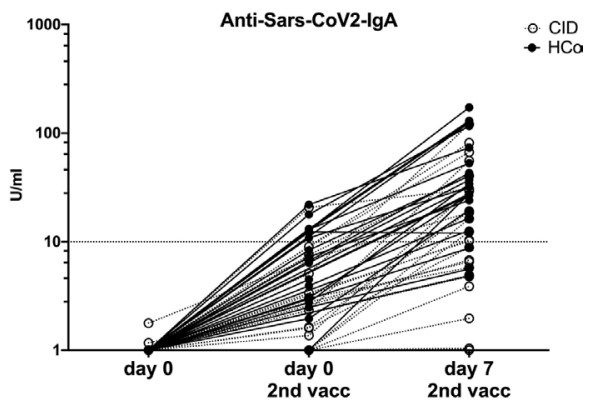

H

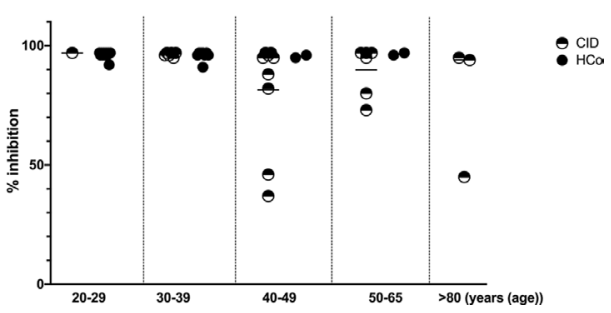

Figure 1 SARS-CoV-2 specific antibodies are detectable in patients and healthy controls. (A) Anti-SARS-CoV-2 IgG antibodies in patients with CID and controls 7 days after secondary immunisation. (B) IgG titres in patients with CID and controls at baseline on the day of the second immunisations and 7 days later. (C) Neutralising activity at 7 days post secondary immunisation. (D) Change in neutralising antibodies from baseline to day 7 after the second immunisation. (E) Anti-SARS-CoV-2 IgA levels 1 week after the second mRNA vaccination in patients and controls. (E) IgA titres at baseline and 7 days after second vaccination. Anti-SARS-CoV2-IgG titres $(\mathrm{G})$ and neutralising capacity $(\mathrm{H})$ in healthy controls and patients by age group 7 days after secondary vaccination. Each symbol represents a single study participant. Bars represent means. Cut-offs for commercial test are displayed as horizontal dashed lines. CID, chronic inflammatory disease; HCo, healthy control.

response. Additionally, no patient on B cell depleting therapy, mycophenolate or cyclophosphamide was included into the study. Especially B cell depleting therapies are known to decrease vaccination response dramatically.

Due to the small cohort, comparison of different therapeutic targets was statistically not feasible. Comparing TNF alpha blockade as the most prevalent therapeutic target in rheumatology as compared with cDMARDs and anti-interleukin 17 blockade showed no significant difference. Obviously, treatment groups were small, and the SD in the TNF blocker group was high. Therefore, generalising from these data might be inappropriate.

Vaccination does not appear to be a major driver of flare ups in patients with CID as none of our cohort showed a significant 
Table 2 Side effects after secondary immunisation in healthy controls and patients with CID as documented 7 days after the vaccination

\begin{tabular}{|c|c|c|c|c|}
\hline \multirow[b]{2}{*}{ Symptoms } & \multicolumn{2}{|c|}{$\begin{array}{l}\text { Healthy donors } \\
\mathrm{n}=38 / 42(\%)\end{array}$} & \multicolumn{2}{|c|}{$\begin{array}{l}\text { Patients } \\
n=26 / 26(\%)\end{array}$} \\
\hline & $\mathrm{N}$ & $\%$ & $\mathrm{~N}$ & $\%$ \\
\hline Local pain at injection side & 25 & 65.8 & 17 & 65.4 \\
\hline Local reddening & 2 & 5.6 & 2 & 7.7 \\
\hline Local swelling & 4 & 11.1 & 4 & 15.4 \\
\hline Fatigue & 16 & 43.2 & 14 & 53.8 \\
\hline Headache & 13 & 35.1 & 10 & 38.5 \\
\hline Fever $>38^{\circ} \mathrm{C}$ & 5 & 13.5 & 0 & 0 \\
\hline Fever $>40^{\circ} \mathrm{C}$ & 0 & 0 & 0 & 0 \\
\hline Lymph node swelling & 4 & 10.8 & 3 & 11.5 \\
\hline Chills & 8 & 21.6 & 1 & 3.8 \\
\hline Arthralgia & 6 & 16.2 & 4 & 15.4 \\
\hline Myalgia & 12 & 31.6 & 11 & 42.3 \\
\hline Other side effects & 7 & 18.4 & 5 & 19.2 \\
\hline Need for NSAIDs & 10 & 26.3 & 9 & 34.6 \\
\hline
\end{tabular}

activation of their inflammatory disease. Mild side effects were only marginally increased, whereas systemic side effects such as fever were reduced in patients with CID compared with healthy

A

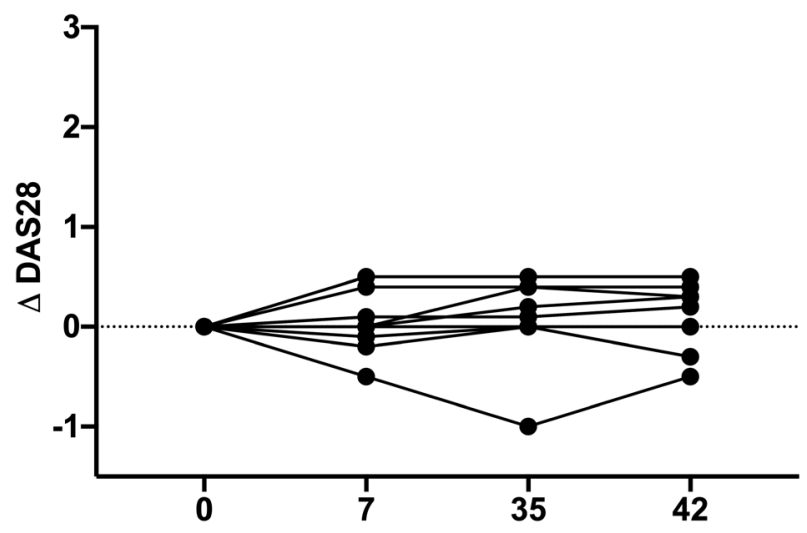

B

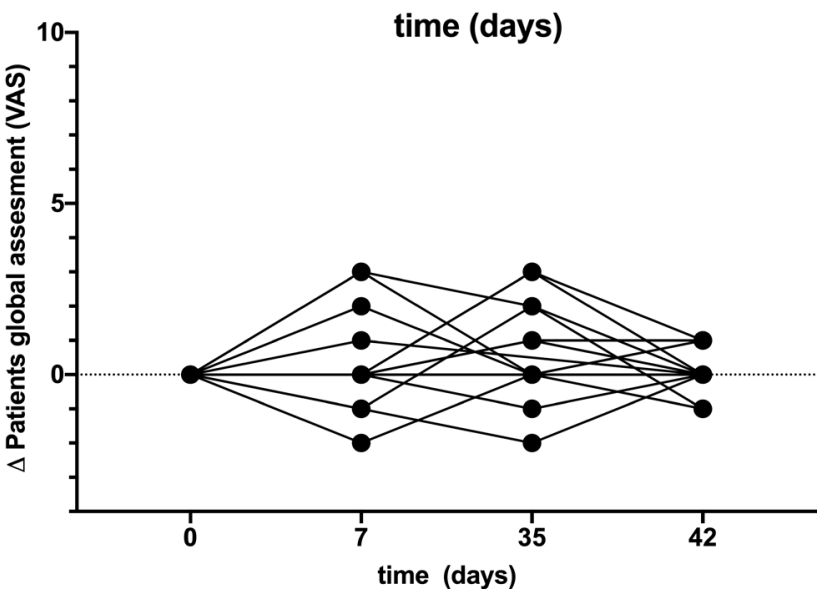

Figure 2 Disease activity does not increase over time after SARSCoV-2 vaccination. (A) Delta DAS28 for patients with inflammatory arthritis during the 42-day study period. (B) Delta patients global assessment in patients with CID from baseline to day 42. Disease activity was assessed before the first and the second immunisation and 7 days after each vaccination. Each symbol represents one patient. CID, chronic inflammatory disease; DAS28, disease activity score 28. controls. These observations may indicate stronger immune reactions in healthy individuals. Such a difference may be due to the younger age of the healthy controls compared with the patients with CID. However, even older controls displayed fever, which was not present in patients. It is also possible that the medication taken by patients with CID is affecting the incidence of systemic side effects.

We are aware that the analysed cohort is small and that our results may be attributable to patient selection. Also, further research is needed to investigate if the differences we observed effect the long-term protection offered by vaccines.

Our data demonstrate for the first time that patients with a selection of immunosuppressive therapies for CID are able to mount an effective immune response after SARS-CoV-2 mRNA vaccination without significant side effects or flares. Thus, we strongly recommend continued vaccination of immunosuppressed patients. However, anti-SARS-CoV-2 antibodies should be monitored in immunosuppressed patients after vaccination, as currently we cannot be certain of antibody titre persistence. The possibility remains that immunosuppressed patients will need a booster (comparable with hepatitis B vaccination) if their antibody titres diminish more rapidly than healthy individuals. Continued monitoring of vulnerable patient groups will be critical in the successful long-term vaccination against SARS-CoV-2.

\section{Author affiliations}

${ }^{1}$ Medical Department I, Department for Rheumatology and Clinical Immunology, University Medical Center Schleswig-Holstein Campus Kiel, Kiel, Schleswig-Holstein, Germany

${ }^{2}$ Department for Internal Medicine I, University Medical Center Schleswig-Holstein

Campus Kiel, Kiel, Schleswig-Holstein, Germany

${ }^{3}$ Institute for Clinical Molecular Biology, Christian-Albrechts-Universitat zu Kiel, Kiel,

Schleswig-Holstein, Germany

${ }^{4}$ Department for Dermatology, University Medical Center Schleswig-Holstein Campus Kiel, Kiel, Schleswig-Holstein, Germany

${ }^{5}$ Department for Rheumatology and Clinical Immunology, University Medical Center Schleswig-Holstein Campus Kiel, Kiel, Schleswig-Holstein, Germany

${ }^{6}$ Department for Pediatrics, University Medical Center Schleswig-Holstein Campus Kiel, Kiel, Schleswig-Holstein, Germany

${ }^{7}$ Institute for Clinical Molecular Biology, Christian-Albrechts-Universität zu Kiel, Kiel, Schleswig-Holstein, Germany

${ }^{8}$ Institute of Clinical Chemistry, University Medical Center Schleswig-Holstein

Campus Kiel, Kiel, Schleswig-Holstein, Germany

${ }^{9}$ Endokrinologikum-Gruppe, Berlin, Germany

${ }^{10}$ medac GmbH, Wedel, Germany

${ }^{11}$ Institute of Clinical Chemistry, Universitätsklinikum Schleswig-Holstein - Campus

Lübeck, Lubeck/Kiel, Germany

${ }^{12}$ Medical Department I, University Medical Center Schleswig-Holstein Campus Kiel, Kiel, Schleswig-Holstein, Germany

Correction notice This article has been corrected sincd it published Online First. Figure 1 has been updated.

Twitter Bimba F Hoyer @rheuma_doktorin

Acknowledgements We would like to thank Ina Martens for perfect technical assistance and Meike Zahnen for organisatory support. We kindly thank Rainer Ahrendt from Medac for providing support on the CPASS test.

Contributors Study design: UMG, BFH, SS and FT. Sample collection: DKB, FT, MS, SG, AS, RZ, JHS, ACL and AF. Experiments and data analysis: UMG, DKB, LV, HMR, MC, $J F, R D H M, C K$, and BFH. Tables and figure: BFH, SG and UMG. Data interpretation: $\mathrm{BFH}$ and UMG. Writing of the manuscript: BFH, UMG, PH and PM. Critical proof reading of the manuscript: all authors.

Funding The project was supported by the Bundesministerium für Blldung und Forschung (BMBF)-funded consortium GAIN and the Deutsche Forschungsgemeinschaft (DFG)-funded excellence cluster 'Precision Medicine in chronic inflammation'.

Competing interests Both BFH and SS received funding from Pfizer and other companies.

Patient consent for publication Not required.

Ethics approval The study was reviewed and approved by the Kiel medical Faculty Ethics Board D 409/21. 
Provenance and peer review Not commissioned; externally peer reviewed.

Data availability statement All data relevant to the study are included in the article or uploaded as supplementary information. Data are under embargo by local authorities if not included into the manuscript.

Supplemental material This content has been supplied by the author(s). It has not been vetted by BMJ Publishing Group Limited (BMJ) and may not have been peer-reviewed. Any opinions or recommendations discussed are solely those of the author(s) and are not endorsed by BMJ. BMJ disclaims all liability and responsibility arising from any reliance placed on the content. Where the content includes any translated material, BMJ does not warrant the accuracy and reliability of the translations (including but not limited to local regulations, clinical guidelines, terminology, drug names and drug dosages), and is not responsible for any error and/or omissions arising from translation and adaptation or otherwise.

Open access This is an open access article distributed in accordance with the Creative Commons Attribution Non Commercial (CC BY-NC 4.0) license, which permits others to distribute, remix, adapt, build upon this work non-commercially, and license their derivative works on different terms, provided the original work is properly cited, appropriate credit is given, any changes made indicated, and the use is non-commercial. See: http://creativecommons.org/licenses/by-nc/4.0/.

ORCID iDs

Robert DH Markewitz http://orcid.org/0000-0003-1676-1085

Bimba F Hoyer http://orcid.org/0000-0002-5252-1719

\section{REFERENCES}

1 Hasseli R, Mueller-Ladner U, Hoyer BF, et al. Older age, comorbidity, glucocorticoid use and disease activity are risk factors for COVID-19 hospitalisation in patients with inflammatory rheumatic and musculoskeletal diseases. RMD Open 2021;7:e001464.

2 Leipe J, Hoyer BF, Iking-Konert C, et al. [SARS-CoV-2 \& rheumatic disease : Consequences of the SARS-COV-2 pandemic for patients with inflammatory rheumatic diseases. A comparison of the recommendations for action of rheumatological societies and risk assessment of different antirheumatic treatments]. Z Rheumatol 2020;79:686-91.

3 Brenner EJ, Ungaro RC, Gearry RB, et al. Corticosteroids, but not TNF antagonists, are associated with adverse COVID-19 outcomes in patients with inflammatory bowel diseases: results from an international registry. Gastroenterology 2020;159:481-91.

4 Gisondi P, Piaserico S, Naldi L, et al. Incidence rates of hospitalization and death from COVID-19 in patients with psoriasis receiving biological treatment: A Northern Italy experience. J Allergy Clin Immunol 2021;147:558-60.

5 Mahil SK, Dand N, Mason KJ, et al. Factors associated with adverse COVID-19 outcomes in patients with psoriasis-insights from a global registry-based study. J Allergy Clin Immunol 2021;147:60-71.

6 Gianfrancesco M, Hyrich KL, Al-Adely S, et al. Characteristics associated with hospitalisation for COVID-19 in people with rheumatic disease: data from the
COVID-19 global rheumatology alliance physician-reported registry. Ann Rheum Dis 2020;79:859-66.

7 Aziz H, Lashkari N, Yoon YC, et al. Effects of coronavirus disease 2019 on solid organ transplantation. Transplant Proc 2020;52:2642-53.

8 Schulze-Koops H, Krueger K, Vallbracht I, et al. Increased risk for severe COVID-19 in patients with inflammatory rheumatic diseases treated with rituximab. Ann Rheum Dis 2021;80:e6.

9 Strangfeld A, Schafer M, Gianfrancesco MA. Factors associated with COVID-19related death in people with rheumatic diseases: results from the COVID-19 global rheumatology alliance physician-reported registry. Ann Rheum Dis 2021.

10 Rubin DT, Abreu MT, Rai V, et al. Management of patients with Crohn's disease and ulcerative colitis during the coronavirus Disease-2019 pandemic: results of an international meeting. Gastroenterology 2020;159:6-13.

11 Schett G, Manger B, Simon D, et al. COVID-19 revisiting inflammatory pathways of arthritis. Nat Rev Rheumatol 2020;16:465-70.

12 Burmester GR, Landewé R, Genovese MC, et al. Adalimumab long-term safety: infections, vaccination response and pregnancy outcomes in patients with rheumatoid arthritis. Ann Rheum Dis 2017;76:414-7.

13 Dugan HL, Henry C, Wilson PC. Aging and influenza vaccine-induced immunity. Cell Immunol 2020;348:103998.

14 Furer V, Rondaan C, Heijstek MW, et al. 2019 update of EULAR recommendations for vaccination in adult patients with autoimmune inflammatory rheumatic diseases. Ann Rheum Dis 2020:79:39-52.

15 García-Agudo R, Aoufi Rabih S, Araque Torres P, et al. Efficacy of a hepatitis B vaccination schedule with two cycles of four double doses of conventional vaccine and four doses of adjuvanted vaccine in chronic kidney disease patients evaluated for renal transplantation. Transplant Proc 2012;44:2532-4.

16 Baden LR, El Sahly HM, Essink B, et al. Efficacy and safety of the mRNA-1273 SARS CoV-2 vaccine. N Engl J Med 2021;384:403-16.

17 Polack FP, Thomas SJ, Kitchin N, et al. Safety and efficacy of the BNT162b2 mRNA Covid-19 vaccine. N Engl J Med 2020;383:2603-15.

18 Voysey M, Clemens SAC, Madhi SA, et al. Safety and efficacy of the ChAdOx 1 $\mathrm{nCoV}-19$ vaccine (AZD1222) against SARS-CoV-2: an interim analysis of four randomised controlled trials in Brazil, South Africa, and the UK. Lancet 2021;397:99-111.

19 Winthrop KL, Bingham CO, Komocsar WJ, et al. Evaluation of pneumococcal and tetanus vaccine responses in patients with rheumatoid arthritis receiving baricitinib: results from a long-term extension trial substudy. Arthritis Res Ther 2019;21:102.

20 Day AL, Winthrop KL, Curtis JR. The effect of disease-modifying antirheumatic drugs on vaccine immunogenicity in adults. Cleve Clin J Med 2020;87:695-703.

21 Taylor SC, Hurst B, Charlton CL, et al. A new SARS CoV-2 dual purpose serology test: highly accurate infection tracing and neutralizing antibody response detection. J Clin Microbio/ 2021. doi:10.1128/JCM.02438-20. [Epub ahead of print: 26 Jan 2021].

22 Walsh EE, Frenck RW, Falsey AR, et al. Safety and immunogenicity of two RNA-based Covid-19 vaccine candidates. N Eng/ J Med 2020;383:2439-50. 